\title{
Voltage sensor inactivation in potassium channels
}

\author{
Robert Bähring *, Jan Barghaan, Regina Westermeier and Jessica Wollberg \\ Institut für Zelluläre und Integrative Physiologie, Zentrum für Experimentelle Medizin, Universitätsklinikum Hamburg-Eppendorf, Hamburg, Germany
}

Edited by:

Gildas Loussouarn, University of

Nantes, France

Reviewed by:

Harley Takatsuna Kurata, University of British Columbia, Canada

Keith Elmslie, AT Still University of

Health Sciences, USA

${ }^{*}$ Correspondence:

Robert Bähring, Institut für Zelluläre und Integrative Physiologie, Zentrum für Experimentelle Medizin,

Universitätsklinikum

Hamburg-Eppendorf, Martinistr. 52,

20246 Hamburg, Germany.

e-mail: r.baehring@

uke.uni-hamburg.de
In voltage-gated potassium (Kv) channels membrane depolarization causes movement of a voltage sensor domain. This conformational change of the protein is transmitted to the pore domain and eventually leads to pore opening. However, the voltage sensor domain may interact with two distinct gates in the pore domain: the activation gate (A-gate), involving the cytoplasmic S6 bundle crossing, and the pore gate (P-gate), located externally in the selectivity filter. How the voltage sensor moves and how tightly it interacts with these two gates on its way to adopt a relaxed conformation when the membrane is depolarized may critically determine the mode of Kv channel inactivation. In certain Kv channels, voltage sensor movement leads to a tight interaction with the P-gate, which may cause conformational changes that render the selectivity filter non-conductive ("P/C-type inactivation"). Other Kv channels may preferably undergo inactivation from pre-open closed-states during voltage sensor movement, because the voltage sensor temporarily uncouples from the A-gate. For this behavior, known as "preferential" closed-state inactivation, we introduce the term "A/C-type inactivation". Mechanistically, P/C- and A/C-type inactivation represent two forms of "voltage sensor inactivation."

Keywords: Kv channels, P/C-type inactivation, closed-state inactivation, U-type inactivation

\section{INTRODUCTION}

Voltage-gated potassium (Kv) channels control excitability and discharge behavior of nerve and muscle cells (Hille, 2001). Kv channel activation may occur in response to strong membrane depolarization, which occurs when action potentials are generated. In this case, the voltage-dependent activation of a potassium conductance critically determines repolarization and afterhyperpolarization (Hodgkin and Huxley, 1952). Alternatively, Kv channels may be activated by subthreshold depolarization, and the resultant potassium conductance may prevent or delay action potential firing (Connor and Stevens, 1971). Notably, many Kv channels undergo inactivation when the membrane is depolarized, meaning that they adopt a non-conducting conformation, which is different from the resting state, and from which the channels can only recover when the membrane is re- or hyperpolarized (Hille, 2001). Whenever such inactivation occurs, the influence of potassium flow on the membrane potential is only transient and provided with refractoriness, which may be of physiological relevance: For instance, the transient nature of a potassium conductance in ventricular cardiomyocytes supports the spikeand-dome morphology of the action potential (Greenstein et al., 2000); in the nervous system brief inactivation of a subthreshold operating dendritic potassium conductance at postsynaptic sites, which normally suppresses dendritic excitation, may lead to local dendritic spike amplification (Hoffman et al., 1997; Magee and Johnston, 1997); and the slow and incomplete recovery of a presynaptic potassium conductance from inactivation may lead to frequency-dependent spike broadening, thereby enhancing calcium entry and transmitter release (Jackson et al., 1991). These are only a few examples showing that $\mathrm{Kv}$ channel inactivation may serve electrical signaling on the cellular level. Due to its critical role in cell physiology, the kinetics, voltage dependence, and structurefunction relationships of $\mathrm{Kv}$ channel inactivation have been the focus of intense research for decades.

Three main mechanisms have been identified so far that may lead to the inactivation of Kv channels (Kurata and Fedida, 2006; Bähring and Covarrubias, 2011). (1) When the cloning of Kv channel genes made detailed structure-function analyses possible, the first inactivation mechanism to be identified was "N-type inactivation"; the name refers to the part of the channel protein involved: A tethered $\mathrm{N}$-terminal inactivation domain of the channel protein plugs the open pore according to a ball-and-chain mechanism (Hoshi et al., 1990); N-type inactivation is usually very fast (on the time scale of few milliseconds). (2) Next, it was recognized that more C-terminal portions of the channel protein also play an important role in $\mathrm{Kv}$ channel inactivation. The fact that the pore domain is involved in this mechanism led to the term "P/Ctype inactivation": Conformational changes at the external pore entrance near the selectivity filter (the P-gate) impair channel conductivity (Hoshi et al., 1991; Yellen et al., 1994). Like N-type inactivation, $\mathrm{P} / \mathrm{C}$-type inactivation has been classically viewed as an open state inactivation mechanism, however, P/C-type inactivation may also occur from closed-states (see below). (3) Finally, some $\mathrm{Kv}$ channels preferably inactivate from closed-states and not from the open state. Although the existence of such "preferential" closed-state inactivation (CSI) was recognized early on, the structural determinants involved have remained a mystery for a long time. It is now thought that temporary uncoupling of the voltage sensor from the $\mathrm{S} 6$ activation gate (A-gate) underlies preferential CSI (Barghaan and Bähring, 2009); P/C-type inactivation; and preferential CSI represent slower processes (usually on the time scale of tens to hundreds of milliseconds). 
Fast N-type inactivation can be abolished by genetic or enzymatic removal of the $\mathrm{N}$-terminal inactivation domain, and experimental application of the isolated inactivation peptide can restore inactivation (Hoshi et al., 1990; Zagotta et al., 1990). N-type inactivation may also be conferred to non- or slowly inactivating channels by their association with auxiliary $\beta$-subunits which for their part possess an N-terminal inactivation domain (Rettig et al., 1994; Wallner et al., 1999; Jerng et al., 2009). By contrast, the slower $\mathrm{P} / \mathrm{C}$-type inactivation and preferential CSI are more intrinsic to the channel. P/C-type inactivation can be prevented by external TEA, elevated external potassium concentrations and by a threonine to valine mutation at the external pore entrance (Choi et al., 1991; Lopez-Barneo et al., 1993). Preferential CSI is critically influenced by mutations located on the internal side of the pore; i.e., the S4-S5 linker and the S6 activation gate (Jerng et al., 1999; Barghaan and Bähring, 2009). Notably, both P/C-type inactivation and preferential CSI of $\mathrm{Kv}$ channels critically depend on conformational changes of the voltage sensor domain (Kurata and Fedida, 2006; Bähring and Covarrubias, 2011). Due to the fact that the voltage sensor is instrumental in both mechanisms, P/Ctype inactivation and preferential CSI may be seen as two forms of "voltage sensor inactivation". Here we provide a brief overview of work, that has demonstrated and mechanistically examined preferential CSI and P/C-type inactivation in the Shaker-related Kv channel subfamilies Kv1-Kv4.

\section{FORMAL DESCRIPTION OF Kv CHANNEL INACTIVATION}

A mechanistic understanding of ion channel function requires the definition of different states which the protein complex may adopt. The formal description is then usually followed by the assignment of structural correlates based on experimental evidence. The basic features of $\mathrm{Kv}$ channel gating, including voltage-dependent activation, pore opening and channel inactivation, may be formally described by the simplified scheme shown in Figure 1A (Scheme $1)$. When the membrane is depolarized $(\Delta V)$ the channels go from a resting state $\left(C_{R}\right)$, via an activated but still closed-state $\left(C^{*}\right)$, to an open state $(\mathrm{O})$. The channels may inactivate either from $\mathrm{C}^{*}(\mathrm{CSI})$ to adopt a closed-inactivated state $\left(\mathrm{I}_{\mathrm{C}}\right)$ or from the open state to reach one of two different but interconnected open-inactivated states $\left(\mathrm{I}_{\mathrm{N}}\right.$ or $\left.\mathrm{I}_{\mathrm{P} / \mathrm{C}}\right)$, representing the mechanisms of $\mathrm{N}$-type and $\mathrm{P} / \mathrm{C}$-type inactivation, respectively. $\mathrm{N}$-type inactivation is thought to favor entry into the $\mathrm{P} / \mathrm{C}$-type inactivated state $\left(\mathrm{I}_{\mathrm{N}, \mathrm{P} / \mathrm{C}}\right)$ due to the prevention of ion flux and the resultant ion deprivation of the pore (Baukrowitz and Yellen, 1995). Scheme 1 does not account for the tetrameric arrangement which allows cooperativity of $\mathrm{Kv}$ channel gating. Also, Scheme 1 assumes that P/C-type inactivation requires channel opening, which represents a classical view but may not be appropriate. A more sophisticated kinetic scheme, suitable for global kinetic modeling (Kaulin et al., 2008), is shown in Figure 1B (Scheme 2). Here, during activation the channels go from a resting state $\left(C_{R}\right)$, via several partially activated closedstates $\left(\mathrm{C}_{1}-\mathrm{C}_{4}\right)$ and an opening-permissive pre-open closed-state $\left(\mathrm{C}_{5}\right)$, to an open state $(\mathrm{O})$. The channels can inactivate from the partially activated and pre-open closed-states to adopt either one of the states $A_{R}-A_{5}$ (these transitions represent preferential CSI), or the channels may adopt one of the inactivated states $\mathrm{P}_{\mathrm{R}}-\mathrm{P}_{6}$ (these transitions represent $\mathrm{P} / \mathrm{C}$-type inactivation). Translated into

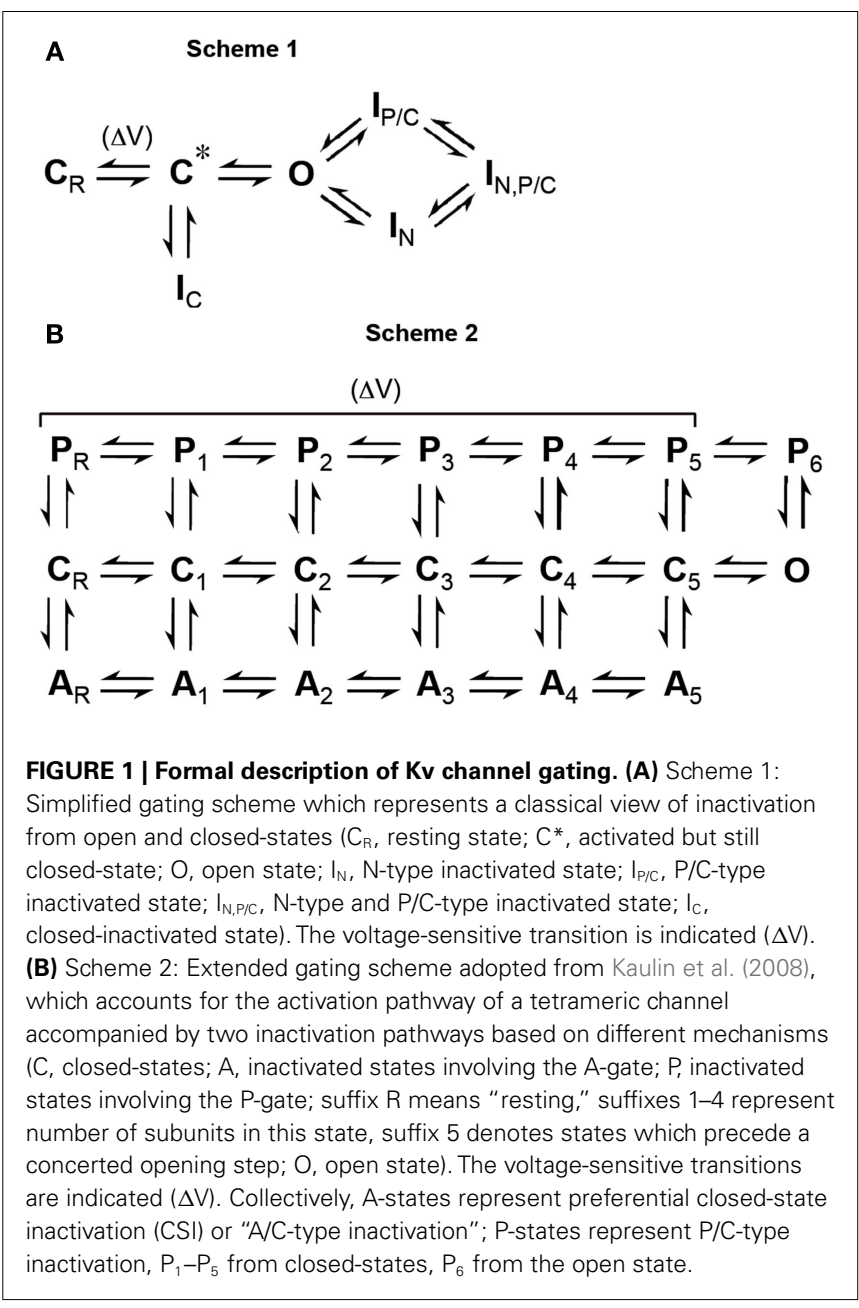

structural terms (see also Figure 2), the majority of transitions from left to right in Scheme 2 are associated with conformational changes of the voltage sensor $(\Delta V$, Figure 1B). When the voltage sensor has adopted an activated conformation in all four subunits (states $\mathrm{C}_{1}-\mathrm{C}_{4}$ ) the channel goes to an opening-permissive conformation $\left(\mathrm{C}_{5}\right)$ from which it can undergo a concerted opening step $(\mathrm{O})$. However, voltage sensor movement may also induce different forms of inactivation, as it either leads to an uncoupling from the A-gate (A-states) or to a tight interaction with the P-gate, thereby causing conformational changes in the selectivity filter which reduce conductivity (P-states). These structural determinants will be discussed in more detail. Notably, Scheme 2 allows P/C-type inactivation from both open and closed-states (see below). Similar to previously suggested models accounting for parallel pathways of P/C-type inactivation and preferential CSI (Klemic et al., 2001; Kurata et al., 2005), Scheme 2 does not account for N-type inactivation (Figure 1B), because it was originally developed for channels in which this form of inactivation plays a negligible role (Kaulin et al., 2008). Also, in the more detailed discussion of preferential CSI and P/C-type inactivation as two forms of voltage sensor inactivation below, we shall leave $\mathrm{N}$-type inactivation unconsidered. We will first discuss indications and the mechanistic basis of preferential CSI in Kv channels, and 
A

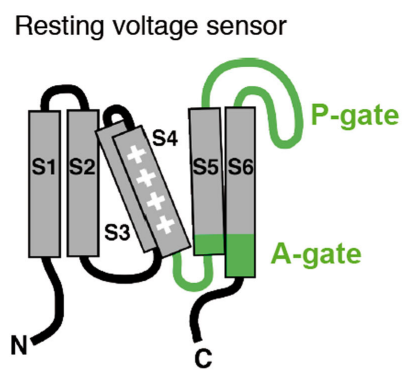

Channel closed

\section{B Activated voltage sensor}

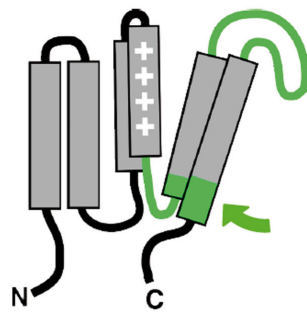

Channel open
C Relaxed voltage sensor

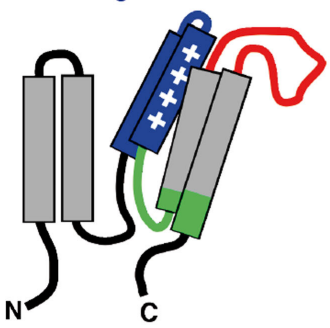

P/C-type inactivation of open channel
D Relaxed voltage sensor

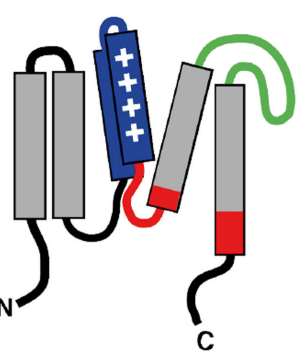

A/C-type inactivation (A-gate uncoupled)
E Relaxed voltage sensor
FIGURE 2 | Models of voltage-dependent activation and voltage sensor inactivation in Kv channels. Cartoons illustrate putative conformations a single Kv channel $\alpha$-subunit may adopt in a subtype-specific manner when the membrane encounters prolonged depolarization. (A) Resting state; the voltage sensor is in its resting position (down) and the channel is closed (S1-S6, transmembrane segments; P-gate, pore gate with selectivity filter; A-gate, activation gate represented by the distal $\mathrm{S} 6$ segment, which is involved in the bundle crossing in the tetramer). (B) Open conducting state; the voltage sensor is activated (up) and the channel is open (green arrow denotes putative opening motion of the A-gate). (C) Open but non-conducting state; the voltage sensor has adopted a relaxed conformation (blue) which tightly interacts with the P-gate rendering the selectivity filter non-conducting (red). (D) Closed-inactivated state with the A-gate uncoupled (red); the voltage sensor has adopted a relaxed conformation (blue), and the A-gate is closed (A/C-type inactivation). (E) Closed-inactivated state with the P-gate in its non-conductive conformation (red); the voltage sensor has adopted a relaxed conformation (blue), the A-gate is still coupled to the voltage sensor but the channel has not opened (P/C-type inactivation of a closed channel). then consider P/C-type inactivation as an alternative reflection of voltage sensor inactivation based on differences in the crosstalk between voltage sensor domain and pore domain.

\section{INDICATIONS OF PREFERENTIAL CSI IN Kv CHANNELS}

The first indication of a cumulative form of inactivation in $\mathrm{Kv}$ channels, which may involve pre-open closed-states, came from recordings done on molluscan neurons (Aldrich, 1981). In this study Aldrich noticed that, after a voltage pulse, the maximum current obtained with a second pulse was less than the current at the end of the first pulse. This finding indicated that, instead of recovery from inactivation, even more inactivation must have occurred during the interpulse interval at a negative voltage. Accordingly, $\mathrm{Kv}$ channels which preferentially inactivate from closed rather than open states show faster and stronger inactivation in response to a series of test pulses rather than to one long pulse. Furthermore, at steady-state Kv channels with preferential CSI show pronounced inactivation at intermediate voltages and less inactivation at more positive voltages where the open probability is increased. Such behavior produces U-shaped inactivation curves, which led to the term "U-type inactivation," with no reference to the structures involved. Kv2.1 (Klemic et al., 1998) and Kv3.1 channels (Klemic et al., 2001) undergo strong cumulative inactivation with repeated brief voltage pulses, and the inactivation curves of these channels exhibit a U-type profile, indicative of preferential CSI. U-type features of steady-state inactivation curves as an indication of preferential CSI have also been observed for N-terminally truncated Shaker channels (Sh $\Delta$; Klemic et al., 2001) and a naturally occurring Kv1.5 N-terminal deletion mutant (Kv1.5 $\Delta$ N209; Klemic et al., 2001; Kurata et al., 2001; Kurata et al., 2002; Kurata et al., 2005).

Computer modeling of non-Shaker A-type potassium currents in neurons from Drosophila (Solc and Aldrich, 1990) and of the transient outward current $\left(I_{\text {to }}\right)$ in ferret ventricular myocytes (Campbell et al., 1993) suggested that inactivation coupled to the voltage-dependent activation pathway of the underlying channels is necessary to reproduce the experimentally observed gating behavior (Ic-state in Scheme 1, A-states in Scheme 2, Figure 1). Moreover, it was observed that prepulse inactivation of the ferret $I_{\text {to }}$ occurred at voltages where no opening was detectable (Campbell et al., 1993). The pioneering study by Campbell and coworkers and the fact that $I_{\text {to }}$ is mainly mediated by members of the Kv 4 subfamily of Kv channels (Dixon et al., 1996), led to a focusing on Kv4 channels to learn more about the mechanism of preferential CSI. It is also important in this context that P/C-type mechanisms play a negligible role in $\mathrm{Kv} 4$ channel inactivation, as external TEA does 
not influence (Jerng and Covarrubias, 1997) and high external potassium concentrations accelerate macroscopic current decay (Jerng and Covarrubias, 1997; Kirichok et al., 1998; Bähring et al., 2001; Shahidullah and Covarrubias, 2003; Kaulin et al., 2008). Notably, the homologous position where a threonine to valine mutation eliminates P/C-type inactivation in ShakerB channels (T449V; Lopez-Barneo et al., 1993), is already occupied by valine in $\mathrm{Kv} 4$ channels.

Just like native $I_{\text {to }}$, recombinant $\mathrm{Kv} 4$ channels in heterologous expression systems show the typical discrepancy between the voltage dependencies of macroscopic inactivation and activation, resulting in almost no overlap of the corresponding curves and a minimal conductance window (Jerng et al., 1999; Bähring et al., 2001; Patel et al., 2004; Dougherty et al., 2008; Kaulin et al., 2008; Barghaan and Bähring, 2009). This is a strong indication of preferential CSI as an intrinsic property of Kv4 channels. Notably, however, the inactivation curves of Kv4 channels do not show Utype features. But this is due to the fact that, even at strongly depolarized membrane voltages, which favor channel opening, occupancy of the open state is only transient in Kv4 channels and they rather tend to close and accumulate in closed-inactivated states (Jerng et al., 1999; Bähring et al., 2001; Barghaan et al., 2008; Kaulin et al., 2008). Thus, these channels exhibit preferential CSI at all relevant voltages. This assertion is based on the finding that the closing step plays a critical role in $\mathrm{Kv} 4$ channel inactivation. Experimentally this has been tested by the use of different permeant ions (high and identical concentrations of one ion species on either side of the membrane): Rubidium ions, which have a longer residency time in the pore than potassium ions not only slow Kv4 deactivation tail currents following brief activation pulses but also macroscopic inactivation during prolonged pulses (Bähring et al., 2001; Shahidullah and Covarrubias, 2003; Barghaan et al., 2008). Also, point mutations, which cause a slowing of deactivation tail current decay, at the same time slow macroscopic inactivation in Kv4 channels (Jerng et al., 1999). Any open state inactivation mechanism would be favored and macroscopic current decay accelerated when channel closing is delayed. Also, recovery from open-inactivated states is usually accompanied by re-opening tail currents before the channels close. The complete absence of such re-opening tail currents when the membrane potential is repolarized after a prolonged voltage pulse sufficiently depolarized to open Kv4 channels (Bähring et al., 2001), also reflects their final accumulation in closed-inactivated states at all relevant voltages.

\section{MECHANISM OF CSI IN Kv4 CHANNELS}

Recent studies have now shed light on what might happen mechanistically during CSI in Kv4 channels. In one study, mutational and thermodynamic analyses of putative coupling domains between the voltage sensor and the A-gate were correlated with CSI kinetics and steady-state inactivation in Kv4.2 channels (Barghaan and Bähring, 2009); in another study a detailed electrophysiological analysis of gating charge movement was performed and the results correlated with Kv4.2 channel inactivation (Dougherty et al., 2008).

Previous findings had already given a hint on the structural correlates that might be responsible for the finding that $\mathrm{Kv}$ channels can stay closed despite voltage sensor movement. For instance, in
N-terminally truncated and L382I point-mutated ShakerB channels enhanced CSI had been observed relative to wild-type (Ayer and Sigworth, 1997). The L382I mutation lies at the S4 end of the S4-S5 linker. Notably, the S4-S5 linker is critically involved in the coupling of the voltage sensor to the A-gate in Kv channels (Lu et al., 2002; Yifrach and MacKinnon, 2002; Long et al., 2005). A physical model of CSI has been suggested (Shin et al., 2004; Barghaan and Bähring, 2009), in which S4-S5 linker residues may fail to make tight contact with their S6 counterparts. Barghaan and Bähring (2009) directly tested this hypothesis by applying scanning mutagenesis and double-mutant cycle analysis to the Kv4.2 S4-S5 linker and the distal S6 segment. Such double-mutant cycle analysis has previously identified pairs of amino acids within these two domains, which, by direct coupling, mediate the voltagedependent opening of the A-gate in Shaker B channels (Yifrach and MacKinnon, 2002). In corresponding Kv4.2 single- and doublemutants, these sites were tested for their involvement in CSI by applying prepulse inactivation protocols. The results of these experiments suggested that pairs of amino acids, one in the S4-S5 linker and the other one in the distal S6 segment, not only interact with each other to open the A-gate, but are dynamic interaction partners in Kv4 channel CSI (Barghaan and Bähring, 2009). It was concluded that Kv4 channel CSI is based on the temporary uncoupling of the voltage sensor from the A-gate.

In voltage-dependent ion channels the movement of gating charge can be influenced by inactivation, as first characterized for voltage-dependent sodium channels (Armstrong and Bezanilla, 1977). In the absence of inactivation gating charges located in S4 may freely move across the membrane electric field (see Figures $2 \mathbf{A}, \mathbf{B}$ ), which can be detected as outward and inward gating currents (Armstrong and Bezanilla, 1973). However, if the return of the voltage sensor to its resting conformation is impeded and slowed ("gating charge immobilization") the detection of gating currents may be constricted or impossible ("loss of gating charge"). This may be the case if the voltage sensor adopts a stable ("relaxed") conformation in response to a prolonged depolarization (see Figures 2C-E). In Kv channels gating charge immobilization has been shown previously for both N-type (Bezanilla et al., 1991; Perozo et al., 1992; Roux et al., 1998) and P/C-type inactivation (Fedida et al., 1996; Olcese et al., 1997). Dougherty et al. (2008) tested whether gating charge immobilization also occurs in Kv4.2 channels known to preferentially undergo CSI. If the dynamic uncoupling model of CSI is correct and conformational changes of the voltage sensor represent the proximate cause of $\mathrm{Kv} 4$ channel CSI, electrophysiological manifestations of inactivation and gating charge immobilization should share the same voltage dependence and kinetics. The experiments by Dougherty et al. (2008) revealed profound gating charge immobilization over a narrow range of negative membrane potentials where CSI is expected to occur. Moreover, the kinetics of the loss of gating charge at positive and recovery of gating charge at negative voltages were identical to the kinetics of ionic current decay and macroscopic recovery from inactivation, respectively. These results showed that the loss of gating charge and the onset of inactivation reflect the same molecular mechanism, and they strongly suggested that the voltage sensor is directly involved in the mechanism of $\mathrm{Kv} 4$ channel CSI. The results also explained why CSI is critically influenced 
by neutralizing positive charges (Skerritt and Campbell, 2007, 2009) or introducing non-native positive charges (Skerritt and Campbell, 2008) in the Kv4.3 S4 segment.

Obviously, CSI in Kv4 channels corresponds to the failure of the voltage sensors to actively open the A-gate. It is not known to date whether uncoupling allows the voltage sensor to adopt its relaxed conformation, or whether this slow conformational change of the voltage sensor actually causes uncoupling. Optical recording techniques, including voltage-clamp fluorimetry, may help to elucidate structural rearrangements during Kv4 channel CSI not accessible by electrophysiological measurements, thereby clarifying the cause-and-effect relationships of voltage sensor relaxation and A-gate uncoupling.

\section{P/C- AND A/C-TYPE INACTIVATION: TWO VARIATIONS OF VOLTAGE SENSOR - PORE DOMAIN CROSSTALK}

$\mathrm{P} / \mathrm{C}$-type inactivation involves conformational changes at the Pgate (Pardo et al., 1992; De Biasi et al., 1993; Lopez-Barneo et al., 1993; Kurata and Fedida, 2006) including the selectivity filter (Ogielska et al., 1995; Panyi et al., 1995; Liu et al., 1996; Kiss and Korn, 1998; Yellen, 1998; Zhou et al., 2001; Kurata and Fedida, 2006; Ahern et al., 2009). Electrostatic interactions involving H-bonds between the pore helix and the selectivity filter have been shown to promote these conformational changes (CorderoMorales et al., 2006, 2007). Notably, gating charge immobilization (see above) has also been observed for P/C-type inactivation (Olcese et al., 1997; Loots and Isacoff, 1998; Larsson and Elinder, 2000; Wang and Fedida, 2001), suggesting that the voltage sensor may also adopt a stable relaxed conformation during prolonged depolarizations (Olcese et al., 1997). These older observations for $\mathrm{P} / \mathrm{C}$-type inactivation are highly similar to the more recent findings for Kv4.2 (Dougherty et al., 2008), a channel in which P/C-type inactivation is known to play a minor role (Kaulin et al., 2008).

Based on the results discussed above, a picture is beginning to evolve where the crosstalk between the voltage sensor domain and the pore domain, which is essential for $\mathrm{Kv}$ channel activation, also represents the structural framework for different manifestations of Kv channel inactivation. The voltage sensor domain has been shown to be able to directly communicate with two distinct gates in the pore domain: the P-gate in the selectivity filter and the A-gate involving the $\mathrm{S} 6$ bundle crossing (Figure 2A; Loots and Isacoff, 2000; Elinder et al., 2001; Lu et al., 2002; Lainé et al., 2003; Webster et al., 2004; Soler-Llavina et al., 2006; Barghaan and Bähring, 2009). When the membrane is depolarized the voltage sensor domain may adopt at least two distinct sets of conformations: First, it moves quickly and makes tight contact with the A-gate to open it (Figure 2B). Then, if the depolarization is sustained, the voltage sensor slowly adopts a more stable (relaxed) conformation (Figures 2C,D). The nature of the relaxed conformation depends on Kv channel subtype: In some Kv channels the voltage sensor may encounter favorable strong interactions with the P-gate stabilizing it in its non-conducting conformation (Loots and Isacoff, 2000; Elinder et al., 2001; Figure 2C). This mechanism corresponds to P/C-type inactivation as discussed above. Other $\mathrm{Kv}$ channels may have P-gates that are not permissive to inactivation, and thus, no inactivation (other than $\mathrm{N}$-type, if a respective inactivation domain is present) will occur (Figure 2B). However, in some $\mathrm{Kv}$ channels with non-inactivating P-gates the voltage sensor domain may interact poorly with the A-gate. In these channels the voltage sensor also starts to move quickly, reaching for the Agate, but the contact may fail or be short-lived due to a "slippery" A-gate. Nevertheless, the voltage sensor of these channels slowly drifts toward a relaxed conformation (Figure 2D). This corresponds to the preferential CSI mechanism discussed above for Kv4 channels. It may also apply to $\mathrm{Kv}$ channels with prominent $\mathrm{U}$-type features of inactivation, like Kv2.1 and Kv3.1.

Although P/C-type inactivation is classically viewed as an open channel inactivation mechanism (see Figures $\mathbf{1 A}$ and $\mathbf{2 C}$ ), recent work has shown that the P-gate may also undergo inactivation when the channel is still closed. Claydon et al. $(2007,2008)$ have performed electrophysiological measurements combined with voltage-clamp fluorimetry to examine whether ShakerIR (N-type inactivation removed) channels may undergo P/C-type inactivation also from closed-states. Acidic $\mathrm{pH}$, which promotes rearrangements at the P-gate, and the Shaker ILT triple-mutant (Smith-Maxwell et al., 1998), which segregates channel opening from voltage-dependent activation by shifting the respective curves apart, were exploited in this study. Conformational changes were inferred by fluorescence changes reported by fluorophores placed on the extracellular S3-S4 loop and an external pore site. The observed signals strongly indicated that the conformational changes involved in P/C-type inactivation also occur when the channels are far from opening (see Figures 1B and 2E). Thus, we encounter a mechanism of CSI, which is of the P/C-type and totally different from the one discussed above for $\mathrm{Kv} 4$ channels. Accordingly, we introduce the term "A/C-type inactivation" to describe the mechanism of preferential CSI found in Kv4 channels, with a direct reference to the structures involved: A/C-type inactivation is nonN-type (i.e., C-type) and involves uncoupling of the voltage sensor from the A-gate. Currently there is no experimental evidence for the simultaneous occurrence of A/C- and P/C-type inactivation in one channel. Notably, however, an elegant study by Kurata et al. (2005) has shown previously that P/C-type and U-type inactivation can co-exist in Kv1.5 channels. It is currently unknown whether an A/C-type or a P/C-type mechanism is responsible for these U-type features.

\section{"INTOXICATION" OF VOLTAGE SENSOR INACTIVATION}

What tools do we have, other than, or in addition to, electrophysiological recordings and voltage-clamp fluorimetry combined with mutational analysis, to study voltage sensor inactivation? Targeting the structures involved with pharmacological tools may be a promising strategy. Intriguingly, gating modifier toxins isolated from tarantula spiders have been shown to act especially on $\mathrm{Kv}$ channels known to exhibit preferential CSI (A/C-type inactivation) including Kv2.1 and Kv4 channels (Swartz and MacKinnon, 1995; Sanguinetti et al., 1997; Diochot et al., 1999; Escoubas et al., 2002; Ebbinghaus et al., 2004). The action of Hanatoxin on Kv2.1/drk1 channels has been mechanistically studied in great detail by Swartz and coworkers: The toxin directly interacts with the voltage sensor, even when it is in the resting (down) conformation; it stabilizes the resting conformation of the voltage sensor, thereby accelerating channel closure and shifting the voltage dependence of activation to more positive potentials (Swartz and 
MacKinnon, 1997a,b; Lee et al., 2003). Based on these findings, it would be of considerable interest to know what the tarantula toxins do to voltage sensor inactivation? As to that, it has been found that the toxins more specific for $\mathrm{Kv} 4$ channels, including Heteropoda toxins, cause a slowing of macroscopic inactivation, an acceleration of recovery from inactivation and a shift of steadystate inactivation curves to more positive potentials (Sanguinetti et al., 1997; Diochot et al., 1999; Escoubas et al., 2002; Ebbinghaus et al., 2004; DeSimone et al., 2009, 2011). These findings are in accordance with a prevention of the relaxed conformation of the voltage sensor because it is stabilized in its resting conformation. Notably, the bacterial KvAP channel, the crystal structure of which has been solved (Lee et al., 2005), also shows clear indications of preferential CSI (Schmidt et al., 2009). As proposed earlier for HCN and Kv4 channels (Shin et al., 2004; Dougherty et al., 2008; Kaulin et al., 2008; Barghaan and Bähring, 2009), an A/C-type inactivation mechanism has been considered in the case of KvAP (Schmidt et al., 2009). Like Kv2.1 and Kv4 channels, KvAP can be modulated by a voltage sensor toxin (VSTx1) via a membraneaccess mechanism and direct binding to the voltage sensor (Lee and MacKinnon, 2004; Ruta and MacKinnon, 2004). However, VSTx1 favors the inactivated state of KvAP, probably by binding to the activated and/or relaxed (up) conformation of the voltage sensor. Thus, although gating modifier toxins appear to be valuable tools for further investigations of voltage sensor inactivation,

\section{REFERENCES}

Ahern, C. A., Eastwood, A. L., Dougherty, D. A., and Horn, R. (2009). An electrostatic interaction between TEA and an introduced pore aromatic drives spring-inthe-door inactivation in Shaker potassium channels. J. Gen. Physiol. 134, 461-469.

Aldrich, R. W. (1981). Inactivation of voltage-gated delayed potassium current in molluscan neurons. A kinetic model. Biophys. J. 36, 519-532.

Armstrong, C. M., and Bezanilla, F. (1973). Currents related to movement of the gating particles of the sodium channels. Nature 242, 459-461.

Armstrong, C. M., and Bezanilla, F. (1977). Inactivation of the sodium channel. II. Gating current experiments. J. Gen. Physiol. 70, 567-590.

Ayer, R. K. Jr., and Sigworth, F. J. (1997). Enhanced closed-state inactivation in a mutant Shaker $\mathrm{K}^{+}$channel. $J$. Membr. Biol. 157, 215-230.

Bähring, R., Boland, L. M., Varghese, A., Gebauer, M., and Pongs, O. (2001). Kinetic analysis of openand closed-state inactivation transitions in human Kv4.2 A-type potassium channels. J. Physiol. (Lond.) 535, 65-81.

Bähring, R., and Covarrubias, M. (2011). Mechanisms of closed-state inactivation in voltage-gated ion channels. J. Physiol. (Lond.) 589, 461-479.

Barghaan, J., and Bähring, R. (2009). Dynamic coupling of voltage sensor and gate involved in closed-state inactivation of Kv4.2 channels. J. Gen. Physiol. 133, 205-224.

Barghaan, J., Tozakidou, M., Ehmke, H., and Bähring, R. (2008). Role of $\mathrm{N}$-terminal domain and accessory subunits in controlling deactivationinactivation coupling of $\mathrm{Kv} 4.2$ channels. Biophys. J. 94, 1276-1294.

Baukrowitz, T., and Yellen, G. (1995). Modulation of $\mathrm{K}^{+}$current by frequency and external $\left[\mathrm{K}^{+}\right]$: a tale of two inactivation mechanisms. Neuron 15, 951-960.

Bezanilla, F., Perozo, E., Papazian, D. M., and Stefani, E. (1991). Molecular basis of gating charge immobilization in Shaker potassium channels. Science 254, 679-683.

Campbell, D. L., Rasmusson, R. L., Qu, Y., and Strauss, H. C. (1993). The calcium-independent transient outward potassium current in isolated ferret right ventricular myocytes. I. Basic characterization and kinetic analysis. J. Gen. Physiol. 101, 571-601.

Choi, K. L., Aldrich, R. W., and Yellen, G. (1991). Tetraethylammonium blockade distinguishes two inactivation mechanisms in voltageactivated $\mathrm{K}^{+}$channels. Proc. Natl. Acad. Sci. U.S.A. 88, 5092-5095.

the questions to be answered first are: Do gating modifier toxins differ in their mode of action, or does the effect of gating modifier toxins critically depend on Kv channel subtype, or both? Doubtlessly, crystallization of a $\mathrm{Kv}$ channel in the absence and presence of a ligand that influences the likelihood of an A/C- or $\mathrm{P} / \mathrm{C}$-type inactivated state would significantly advance structural analysis of voltage sensor inactivation.

\section{CONCLUDING REMARKS}

The purpose of this review was to focus on intrinsic mechanisms of inactivation in $\mathrm{Kv}$ channels that involve conformational changes of the voltage sensor (i.e., voltage sensor inactivation). In particular, we pointed out that subtype-specific variations in the crosstalk between voltage sensor domain and pore domain play a decisive role for the mode of $\mathrm{Kv}$ channel inactivation. Furthermore, in order to up-date current nomenclature of inactivation mechanisms in a useful manner, we introduced the term "A/C-type inactivation." Based on the finding that $\mathrm{P} / \mathrm{C}$-type inactivation can also occur from closed-states, the term "CSI" for a dynamic coupling between voltage sensor and A-gate seems no longer appropriate.

\section{ACKNOWLEDGMENTS}

Our research on $\mathrm{Kv}$ channel gating mechanisms is supported by grant Ba 2055/1-2 from the Deutsche Forschungsgemeinschaft to Robert Bähring.

Claydon, T. W., Kehl, S. J., and Fedida, D. (2008). Closed-state inactivation induced in KV1 channels by extracellular acidification. Channels (Austin) 2, 139-142.

Claydon, T. W., Vaid, M., Rezazadeh, S. Kwan, D. C., Kehl, S. J., and Fedida, D. (2007). A direct demonstration of closed-state inactivation of $\mathrm{K}^{+}$channels at low pH. J. Gen. Physiol. 129, 437-455.

Connor, J. A., and Stevens, C. F. (1971). Prediction of repetitive firing behaviour from voltage clamp data on an isolated neuron soma. J. Physiol. (Lond.) 213, 31-53.

Cordero-Morales, J. F., Cuello, L. G., Zhao, Y., Jogini, V., Cortes, D. M., Roux, B., and Perozo, E. (2006). Molecular determinants of gating at the potassium-channel selectivity filter. Nat. Struct. Mol. Biol. 13, 311-318.

Cordero-Morales, J. F., Jogini, V., Lewis, A., Vasquez, V., Cortes, D. M., Roux, B., and Perozo, E. (2007). Molecular driving forces determining potassium channel slow inactivation. Nat. Struct. Mol. Biol. 14 1062-1069.

De Biasi, M., Hartmann, H. A., Drewe, J. A., Taglialatela, M., Brown, A. M., and Kirsch, G. E. (1993). Inactivation determined by a single site in $\mathrm{K}^{+}$ pores. Pflügers Arch. 422, 354-363.

DeSimone, C. V., Lu, Y., Bondarenko, V. E., and Morales, M. J. (2009). S3b amino acid substitutions and ancillary subunits alter the affinity of Heteropoda venatoria toxin 2 for Kv4.3. Mol. Pharmacol. 76, 125-133.

DeSimone, C. V., Zarayskiy, V. V., Bondarenko, V. E., and Morales, M. J. (2011). Heteropoda toxin 2 interaction with Kv4.3 and Kv4.1 reveals differences in gating modification. Mol. Pharmacol. 80, 345-355.

Diochot, S., Drici, M. D., Moinier, D., Fink, M., and Lazdunski, M. (1999). Effects of phrixotoxins on the Kv4 family of potassium channels and implications for the role of Itol in cardiac electrogenesis. Br. J. Pharmacol. 126, 251-263.

Dixon, J. E., Shi, W., Wang, H. S., McDonald, C., Yu, H., Wymore, R. S., Cohen, I. S., and McKinnon, D. (1996). Role of the Kv4.3 K+ channel in ventricular muscle. A molecular correlate for the transient outward current. Circ. Res. 79, 659-668.

Dougherty, K., De Santiago-Castillo, J. A., and Covarrubias, M. (2008). Gating charge immobilization in Kv4.2 channels: the basis of closed-state inactivation. J. Gen. Physiol. 131, 257-273.

Ebbinghaus, J., Legros, C., Nolting, A., Guette, C., Celerier, M. L., Pongs, O., and Bähring, R. (2004). Modulation of Kv4.2 channels by a peptide isolated from the venom of the giant bird-eating tarantula Theraphosa leblondi. Toxicon 43, 923-932. 
Elinder, F., Männikkö, R., and Larsson, H. P. (2001). S4 charges move close to residues in the pore domain during activation in a K channel. J. Gen. Physiol. 118, 1-10.

Escoubas, P., Diochot, S., Celerier, M. L., Nakajima, T., and Lazdunski, M. (2002). Novel tarantula toxins for subtypes of voltage-dependent potassium channels in the Kv2 and Kv4 subfamilies. Mol. Pharmacol.62, 48-57.

Fedida, D., Bouchard, R., and Chen, F. S. (1996). Slow gating charge immobilization in the human potassium channel Kv1.5 and its prevention by 4-aminopyridine. J. Physiol. (Lond.) 494, 377-387.

Greenstein, J. L., Wu, R., Po, S., Tomaselli, G. F., and Winslow, R. L. (2000). Role of the calciumindependent transient outward current Itol in shaping action potential morphology and duration. Circ. Res. 87, 1026-1033.

Hille, B. (2001). Ion Channels of Excitable Membranes. Sunderland, MA: Sinauer Associates, Inc.

Hodgkin, A. L., and Huxley, A. F. (1952). A quantitative description of membrane current and its application to conduction and excitation in nerve. J. Physiol. (Lond.) 117, 500-544.

Hoffman, D. A., Magee, J. C., Colbert, C. M., and Johnston, D. (1997). $\mathrm{K}^{+}$ channel regulation of signal propagation in dendrites of hippocampal pyramidal neurons. Nature 387 , 869-875.

Hoshi, T., Zagotta, W. N., and Aldrich, R. W. (1990). Biophysical and molecular mechanisms of Shaker potassium channel inactivation. Science 250, 533-538.

Hoshi, T., Zagotta, W. N., and Aldrich, R. W. (1991). Two types of inactivation in Shaker $\mathrm{K}^{+}$channels: effects of alterations in the carboxy-terminal region. Neuron 7, 547-556.

Jackson, M. B., Konnerth, A., and Augustine, G. J. (1991). Action potential broadening and frequency-dependent facilitation of calcium signals in pituitary nerve terminals. Proc. Natl. Acad. Sci. U.S.A. 88, 380-384.

Jerng, H. H., and Covarrubias, M. (1997). $\mathrm{K}^{+}$channel inactivation mediated by the concerted action of the cytoplasmic N- and C-terminal domains. Biophys. J. 72, 163-174.

Jerng, H. H., Dougherty, K., Covarrubias, M., and Pfaffinger, P. J. (2009). A novel N-terminal motif of dipeptidyl peptidase-like proteins produces rapid inactivation of KV4.2 channels by a pore-blocking mechanism. Channels (Austin) 3, 448-461.
Jerng, H. H., Shahidullah, M., and Covarrubias, M. (1999). Inactivation gating of $\mathrm{Kv} 4$ potassium channels: molecular interactions involving the inner vestibule of the pore. $J$. Gen. Physiol. 113, 641-660.

Kaulin, Y. A., De Santiago-Castillo, J. A., Rocha, C. A., and Covarrubias, M. (2008). Mechanism of the modulation of Kv4:KChIP-1 channels by external $\mathrm{K}^{+}$. Biophys. J. 94, 1241-1251.

Kirichok, Y. V., Nikolaev, A. V., and Krishtal, O. A. (1998). $\left[\mathrm{K}^{+}\right]$out accelerates inactivation of Shalchannels responsible for A-current in rat CA1 neurons. Neuroreport 9, 625-629.

Kiss, L., and Korn, S. J. (1998). Modulation of C-type inactivation by $\mathrm{K}^{+}$ at the potassium channel selectivity filter. Biophys. J. 74, 1840-1849.

Klemic, K. G., Kirsch, G. E., and Jones, S. W. (2001). U-type inactivation of Kv3.1 and Shaker potassium channels. Biophys. J. 81, 814-826.

Klemic, K. G., Shieh, C. C., Kirsch, G. E., and Jones, S. W. (1998). Inactivation of Kv2.1 potassium channels. Biophys. J. 74, 1779-1789.

Kurata, H. T., Doerksen, K. W., Eldstrom, J. R., Rezazadeh, S., and Fedida, D. (2005). Separation of $\mathrm{P} / \mathrm{C}$ - and U-type inactivation pathways in Kv1.5 potassium channels. J. Physiol. (Lond.) 568, 31-46.

Kurata, H. T., and Fedida, D. (2006). A structural interpretation of voltagegated potassium channel inactivation. Prog. Biophys. Mol. Biol. 92, 185-208.

Kurata, H. T., Soon, G. S., Eldstrom, J. R., Lu, G. W., Steele, D. F., and Fedida, D. (2002). Amino-terminal determinants of $\mathrm{U}$-type inactivation of voltage-gated $\mathrm{K}^{+}$channels. J. Biol. Chem. 277, 29045-29053.

Kurata, H. T., Soon, G. S., and Fedida, D. (2001). Altered state dependence of C-type inactivation in the long and short forms of human Kv1.5. J. Gen. Physiol. 118, 315-332.

Lainé, M., Lin, M. C., Bannister, J. P., Silverman, W. R., Mock, A. F., Roux, B., and Papazian, D. M. (2003). Atomic proximity between S4 segment and pore domain in Shaker potassium channels. Neuron 39, 467-481.

Larsson, H. P., and Elinder, F. (2000). A conserved glutamate is important for slow inactivation in $\mathrm{K}^{+}$channels. Neuron 27, 573-583.

Lee, H. C., Wang, J. M., and Swartz, K. J. (2003). Interaction between extracellular Hanatoxin and the resting conformation of the voltage-sensor paddle in Kv channels. Neuron 40, 527-536.
Lee, S. Y., Lee, A., Chen, J., and MacKinnon, R. (2005). Structure of the KvAP voltage-dependent $\mathrm{K}^{+}$channel and its dependence on the lipid membrane. Proc. Natl. Acad. Sci. U.S.A. 102, 15441-15446.

Lee, S. Y., and MacKinnon, R. (2004). A membrane-access mechanism of ion channel inhibition by voltage sensor toxins from spider venom. Nature 430, 232-235.

Liu, Y., Jurman, M. E., and Yellen, G. (1996). Dynamic rearrangement of the outer mouth of a $\mathrm{K}^{+}$channel during gating. Neuron 16, 859-867.

Long, S. B., Campbell, E. B., and MacKinnon, R. (2005). Voltage sensor of Kv1.2: structural basis of electromechanical coupling. Science 309, 903-908.

Loots, E., and Isacoff, E. Y. (1998). Protein rearrangements underlying slow inactivation of the Shaker $\mathrm{K}^{+}$channel. J. Gen. Physiol. 112, 377-389.

Loots, E., and Isacoff, E. Y. (2000). Molecular coupling of $\mathrm{S} 4$ to a $\mathrm{K}^{+}$channel's slow inactivation gate. J. Gen. Physiol. 116, 623-636.

Lopez-Barneo, J., Hoshi, T., Heinemann, S. H., and Aldrich, R. W. (1993). Effects of external cations and mutations in the pore region on Ctype inactivation of Shaker potassium channels. Receptors Channels 1 , 61-71.

Lu,Z., Klem, A. M., and Ramu, Y. (2002). Coupling between voltage sensors and activation gate in voltage-gated $\mathrm{K}^{+}$channels. J. Gen. Physiol. 120, 663-676.

Magee, J. C., and Johnston, D. (1997). A synaptically controlled, associative signal for Hebbian plasticity in hippocampal neurons. Science 275, 209-213.

Ogielska, E. M., Zagotta, W. N., Hoshi, T., Heinemann, S. H., Haab, J., and Aldrich, R. W. (1995). Cooperative subunit interactions in C-type inactivation of K channels. Biophys. J. 69, 2449-2457.

Olcese, R., Latorre, R., Toro, L., Bezanilla, F., and Stefani, E. (1997). Correlation between charge movement and ionic current during slow inactivation in Shaker $\mathrm{K}^{+}$channels. J. Gen. Physiol. 110, 579-589.

Panyi, G., Sheng, Z., and Deutsch, C. (1995). C-type inactivation of a voltage-gated $\mathrm{K}^{+}$channel occurs by a cooperative mechanism. Biophys. J. 69, 896-903.

Pardo, L. A., Heinemann, S. H., Terlau, H., Ludewig, U., Lorra, C., Pongs, O., and Stühmer, W. (1992). Extracellular $\mathrm{K}^{+}$specifically modulates a rat brain $\mathrm{K}^{+}$channel. Proc. Natl. Acad. Sci. U.S.A. 89, 2466-2470.
Patel, S. P., Parai, R., and Campbell, D. L. (2004). Regulation of Kv4.3 voltage-dependent gating kinetics by KChIP2 isoforms. J. Physiol. (Lond.) 557, 19-41.

Perozo, E., Papazian, D. M., Stefani, E., and Bezanilla, F. (1992). Gating currents in Shaker $\mathrm{K}^{+}$channels. Implications for activation and inactivation models. Biophys. J. 62, 160-171.

Rettig, J., Heinemann, S. H., Wunder, F., Lorra, C., Parcej, D. N. Dolly, J. O., and Pongs, O. (1994). Inactivation properties of voltagegated $\mathrm{K}^{+}$channels altered by presence of $\beta$-subunit. Nature 369 , 289-294.

Roux, M. J., Olcese, R., Toro, L., Bezanilla, F., and Stefani, E. (1998). Fast inactivation in Shaker $\mathrm{K}^{+}$channels. Properties of ionic and gating currents. J. Gen. Physiol. 111, 625-638.

Ruta, V., and MacKinnon, R. (2004). Localization of the voltage-sensor toxin receptor on KvAP. Biochemistry 43, 10071-10079.

Sanguinetti, M. C., Johnson, J. H., Hammerland, L. G., Kelbaugh, P. R., Volkmann, R. A., Saccomano, N. A., and Mueller, A. L. (1997). Heteropoda toxins: peptides isolated from spider venom that block Kv4.2 potassium channels. Mol. Pharmacol. 51, 491-498.

Schmidt, D., Cross, S. R., and MacKinnon, R. (2009). A gating model for the archeal voltage-dependent $\mathrm{K}^{+}$channel KvAP in DPhPC and POPE:POPG decane lipid bilayers. $J$. Mol. Biol. 390, 902-912.

Shahidullah, M., and Covarrubias, M. (2003). The link between ion permeation and inactivation gating of $\mathrm{Kv} 4$ potassium channels. Biophys. J. 84, 928-941.

Shin, K. S., Maertens, C., Proenza, C., Rothberg, B. S., and Yellen, G. (2004). Inactivation in HCN channels results from reclosure of the activation gate: desensitization to voltage. Neuron 41, 737-744.

Skerritt, M. R., and Campbell, D. L. (2007). Role of S4 positively charged residues in the regulation of Kv4.3 inactivation and recovery. Am. J. Physiol. Cell Physiol. 293, C906C914.

Skerritt, M. R., and Campbell, D. L. (2008). Non-native R1 substitution in the S4 domain uniquely alters Kv4.3 channel gating. PLoS ONE 3, e3773. doi:10.1371/journal.pone.0003773

Skerritt, M. R., and Campbell, D. L. (2009). Contribution of electrostatic and structural properties of Kv4.3 $\mathrm{S} 4$ arginine residues to the regulation 
Bähring et al.

Voltage sensor inactivation

of channel gating. Biochim. Biophys. Alta 1788, 458-469.

Smith-Maxwell, C. J., Ledwell, J. L., and Aldrich, R. W. (1998). Uncharged S4 residues and cooperativity in voltage-dependent potassium chanhel activation. J. Gen. Physiol. 111, 421-439.

Sole, C. K., and Aldrich, R. W. (1990). Gating of single non-Shaker Atype potassium channels in larval Drosophila neurons. J. Gen. Physiol. 96, 135-165.

Soler-Llavina, G. J., Chang, T. H., and Swartz, K. J. (2006). Functional interactions at the interface between voltage-sensing and pore domains in the Shaker Kv channel. Neuron 52, 623-634.

Swartz, K. J., and MacKinnon, R. (1995). An inhibitor of the Kv2.1 potassium channel isolated from the venom of a Chilean tarantula. Neuron 15, 941-949.

Swartz, K. J., and MacKinnon, R. (1997a). Hanatoxin modifies the gating of a voltage-dependent $\mathrm{K}^{+}$ channel through multiple binding sites. Neuron 18, 665-673.

Swartz, K. J., and MacKinnon, R. (1997b). Mapping the receptor site for hanatoxin, a gating modifier of voltage- dependent $\mathrm{K}^{+}$channels. Neuron 18, 675-682.

Wallner, M., Meera, P., and Too, L. (1999). Molecular basis of fast inctivation in voltage and $\mathrm{Ca}^{2+}$-activa ted $\mathrm{K}^{+}$channels: a transmembrane $\beta$-subunit homolog. Proc. Natl. Acid. Sci. U.S.A. 96, 4137-4142.

Wang, Z., and Fedida, D. (2001). Gating charge immobilization caused by the transition between inactivated states in the Kv1.5 channel. Biophys. J. 81, 2614-2627.

Webster, S. M., Del Camino, D., Dekker, J. P., and Yellen, G. (2004). Intracellular gate opening in Shaker $\mathrm{K}^{+}$channoels defined by high-affinity metal bridges. Nature 428, 864-868.

Helen, G. (1998). The moving parts of voltage-gated ion channels. Q. Rev. Biophys. 31, 239-295.

Yellen, G., Sodickson, D., Chen, T. Y., and Jurman, M. E. (1994). An engineered cysteine in the external mouth of a $\mathrm{K}^{+}$channel allows inactivation to be modulated by metal binding. Biophys. J. 66, 1068-1075.

Yifrach, O., and MacKinnon, R. (2002). Energetics of pore opening in a voltage-gated $\mathrm{K}^{+}$channel. Cell 111, 231-239.

Zagotta, W. N., Hoshi, T., and Aldrich, R. W. (1990). Restoration of inactivetimon in mutants of Shaker potassium channels by a peptide derived from ShR. Science 250, 568-571.

Chou, Y., Morais-Cabral, J. H., Kaufman, A., and MacKinnon, R. (2001). Chemistry of ion coordination and hydration revealed by a $\mathrm{K}^{+}$channelFab complex at $2.0 \AA$ resolution. Nature 414, 43-48.

Conflict of Interest Statement: The authors declare that the research was conducted in the absence of any commercial or financial relationships that could be construed as a potential conflick of interest.

Received: 16 March 2012; accepted: 04 May 2012; published online: 23 May 2012.

Citation: Bähring R, Barghaan J, Westermeier $R$ and Wollberg $J$ (2012) Voltage sensor inactivation in potassium channels. Front. Pharmacol. 3:100. do: 10.3389/fphar.2012.00100

This article was submitted to Frontiers in Pharmacology of Ion Channels and Channelopathies, a specialty of Frontiers in Pharmacology.

Copyright (c) 2012 Bähring, Barghaan, Westermeier and Wollberg. This is an open-access article distributed under the terms of the Creative Commons Attribion Non Commercial License, which permit non-commercial use, distribution, and reproduction in other forums, provide the original authors and source are credited.

Frontiers in Pharmacology | Pharmacology of Ion Channels and Channelopathies

May 2012 | Volume 3 | Article 100 | 8 